\title{
ESTRIDOR INSPIRATORIO EN NEONATO POR NEUROBLASTOMA CERVICAL. DESCRIPCIÓN DE UN CASO
}

\section{Inspiratory stridor in neonate by cervical neuroblastoma. A case report}

\author{
María Rosario GÓMEZ-GONZÁLEZ1'; José Antonio DÍAZ-MANZANO² \\ ${ }^{1}$ Hospital General Universitario Reina Sofía. Servicio Otorrinolaringología. Murcia. España. \\ ${ }^{2}$ Hospital Clínico Universitario Virgen de la Arrixaca. Servicio Otorrinolaringología Universidad de Murcia. Departamento \\ de Otorrinolaringología. Murcia. España.
}

Correspondencia: charo.gomez.gonzalez@gmail.com

Fecha de recepción: 24 de julio de 2017

Fecha de aceptación: 18 de agosto de 2017

Fecha de publicación: 20 de agosto de 2017

Fecha de publicación del fascículo: 1 de marzo de 2018

Conflicto de intereses: Los autores declaran no tener conflictos de intereses

Imágenes: Los autores declaran haber obtenido las imágenes con el permiso de los pacientes

Política de derechos y autoarchivo: se permite el autoarchivo de la versión post-print (SHERPA/RoMEO)

Licencia CC BY-NC-ND. Licencia Creative Commons Atribución-NoComercial-SinDerivar 4.0 Internacional

Universidad de Salamanca. Su comercialización está sujeta al permiso del editor

RESUMEN

PALABRAS CLAVE

SUMMARY

KEYWORDS
Introducción: El neuroblastoma es el tumor sólido más frecuente en la infancia, sin embargo, su localización cervical es rara. Puede presentarse como una masa asintomática o producir estridor, disnea o dificultad para la deglución. Descripción: Paciente varón de un mes de vida que presenta estridor inspiratorio. En la RM se observa una masa compatible con malformación linfática quística unilocular en espacios carotídeo y prevertebral derechos que comprime la vía aérea. El diagnóstico definitivo fue de neuroblastoma tras escisión quirúrgica. Discusión: En la RM el neuroblastoma y las malformaciones linfáticas presentan patrones similares. Este caso muestra la importancia de un correcto diagnóstico diferencial y manejo de las masas cervicales en neonatos.

neuroblastoma; estridor inspiratorio; masa cervical; neonato

Introduction: Neuroblastoma is the solid tumor more frequent in the infancy, although, his cervical location is rare. It may present as a asymptomatic mass or produce stridor, dyspnea o difficulty in swallowing. Description: Patient of a one month old, male reporting inspiratory stridor. RM showed a mass compatible with an unilocular cystic lymphatic malformation in right carotid and prevertebral spaces with airway compromise. The definitive diagnosis was of neuroblastoma after surgical excision. Discussion: In RM neuroblastoma and lymphatic malformations present similar patterns. This case shows the importance of a correct differential diagnosis and approach of cervical masses in newborns.

neuroblastoma; inspiratory stridor; cervical mass; newborn. 


\section{INTRODUCCIÓN}

El estridor inspiratorio es un hallazgo importante en la exploración que implica obstrucción de la vía aérea y requiere una inmediata y adecuada evaluación de la etiología subyacente. En el recién nacido la laringomalacia es la causa más frecuente de estridor inspiratorio. Otras causas como anomalías cardiovasculares, parálisis de cuerda vocal, malformaciones linfáticas, tumores sólidos etc., son menos comunes, pero presentan riesgo de obstrucción completa de la vía aérea. Por lo tanto, se requiere un examen exhaustivo e inicio precoz de un diagnóstico diferencial completo [1].

El neuroblastoma es el tumor sólido más frecuente en la infancia, representando más del $30 \%$ de cánceres infantiles. La edad media de diagnóstico son los 15 meses, apareciendo en la mayoría de los casos antes de los 6 años. Su localización y características histopatológicas pueden variar. Puede aparecer en cualquier localización a lo largo del sistema nervioso simpático, siendo más frecuente abdominal (glándulas suprarrenales) [2]. Sin embargo, el neuroblastoma cervical primario es raro, representando entre el $2 \%$ y el $6 \%$ de todos los neuroblastomas. El neuroblastoma cervical habitualmente se presenta con estridor, disnea y dificultad para la deglución [3].

\section{DESCRIPCIÓN}

Presentamos el caso de un varón de un mes, sin antecedentes obstétricos de interés, que ingresó por disnea y estridor inspiratorio de 15 días de evolución que aumentaba con el llanto provocando un descenso de hasta $80 \%$ de la saturación de oxígeno. No presentaba fiebre ni dificultad para la alimentación oral.

En la exploración destacó polipnea y tiraje con auscultación cardiopulmonar normal. En la fibrolaringoscopia se apreció un abombamiento de superficie lisa y regular desde pared posterolateral derecha rinofaríngea hasta seno piriforme homolateral, comprometiendo la vía aérea y limitando la abducción del aritenoides derecho (Figura 1). Se realizó una resonancia magnética (RM) cervicotorácica (Figura 2) en la que se apreció una lesión hipointensa en T1 e hiperintensa en T2 en región parafaríngea derecha de $52 \times 37 \times 20 \mathrm{~mm}$ ocupando espacio prevertebral y carotídeo derechos y ejerciendo efecto masa sobre la luz faríngea (calibre mínimo de $1,8 \mathrm{~mm}$ ) con una extensión craneocaudal desde C1 hasta D2. Todo ello era sugestivo de malformación linfática quística unilocular, o quiste de segundo arco branquial como segunda opción. Ante estos hallazgos, se planeó un abordaje inicial mediante aspirado y escleroterapia ecoguiados, no siendo posible por encontrar un contenido de muy alta densidad o sólido. Se realizó, por lo tanto, una cervicotomía y extirpación de una masa sólida parafaríngea derecha de superficie lisa y bien delimitada.

El resultado anatomopatológico fue de neuroblastoma pobremente diferenciado.

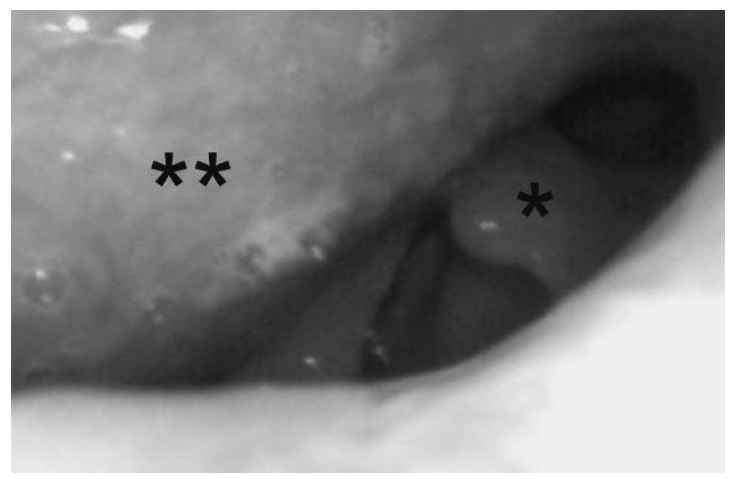

Figura 1. Imagen de fibrolaringoscopia en la que se observa abombamiento de la pared faríngea posterolateral derecha $\left(^{* \star}\right)$. Aritenoides izquierdo $\left({ }^{*}\right)$.

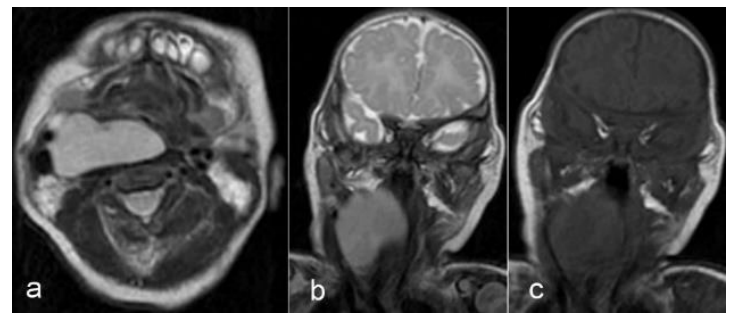

Figura 2. Imagen de RM en la que aparece una masa prevertebral que ocupa el espacio parafaríngeo derecho ocluyendo la luz laríngea, hipointensa en T1 e hiperintensa en T2. (a) Corte axial en T2; (b) corte coronal en T2; (c) corte coronal en $\mathrm{T} 1$.

\section{DISCUSIÓN}

El diagnóstico diferencial de masas cervicales congénitas incluye: tumores vasculares, malformaciones linfáticas, quistes branquiales, defectos cervicales del tubo neural, tejido neuroglial heterotópico, así como tumores sólidos, incluyendo teratomas, neuroblastomas, tumores rabdoides o sarcomas [4].

Muchos son asintomáticos y son descubiertos incidentalmente por los pacientes o en el examen físico, mientras otros precisan de atención médica precoz porque producen efecto 
masa, compromiso de la vía aerodigestiva, fiebre o síntomas relacionados con infección aguda, enfermedad inflamatoria, dolor o deformidades estéticas [5].

En el caso presentado, el paciente comenzó con estridor inspiratorio al mes de vida, sin síntomas de infección, por lo que se sospechó de patología obstructiva. Para realizar el diagnóstico diferencial nos ayudamos de una prueba de imagen. Tanto la tomografía computarizada (TC) como la RM podrían ser usadas en este caso. Ambas pueden evaluar con precisión la localización, el tamaño de la masa, el encasillamiento vascular y la resecabilidad del tumor. Decidimos realizar una RM para evitar la radiación al paciente.

En los últimos años, la RM ha sido considerada la prueba más útil para el estadiaje del neuroblastoma. En la RM el tumor es típicamente heterogéneo con patrones variables de realce, con baja intensidad en T1 y alta intensidad en T2. Sin embargo, hay otros tumores que dan un patrón similar en la RM, como es el caso de malformaciones linfáticas. Los linfangiomas no complicados habitualmente aparecen con señal hipointensa en T1 y marcada hiperintensidad en T2, que puede variar dependiendo del contenido en proteínas y la presencia de productos sanguíneos [5-7].

\section{CONCLUSIONES}

Con este caso se pretende destacar la importancia de establecer un correcto diagnóstico diferencial ante una masa cervical en periodo neonatal, que puede requerir diferentes tratamientos de forma urgente dependiendo de su diagnóstico. Ante la sospecha de una malformación linfática parafaríngea el tratamiento con menor morbilidad sería la escleroterapia o el drenaje mediante abordaje transoral, sin embargo, el tratamiento de elección ante una masa sólida es la escisión quirúrgica. En nuestro caso, realizamos ambos procedimientos en el mismo acto quirúrgico, previendo ambos diagnósticos diferenciales, eliminando así la necesidad de una segunda anestesia general, disminuyendo el tiempo de resolución del problema y evitando la necesidad de una traqueotomía [8, 9].

\section{BIBLIOGRAFÍA}

1. Posod A, Griesmaier E, Brunner A, Pototschnig C, Trawöger KiechlKohlendorfer $U$. An unusual cause of inspiratory stridor in the newborn: congenital pharyngeal teratoma. A case report. BMC Pediatr. 2016;5:16:1.

2. Csanády M, Vass G, Bartyik K, Majoros V, Rovó L. Multidisciplinary management of cervical neuroblastoma in infants. Int J Pediatr Otorhinolaryngol. 2014;78(12):2103-6.

3. Okazaki T, Ohshita M, Furukawa M, Ikeda K, Ozaki Y, Lane GJ, et al. Retropharyngeal neuroblastoma in a neonate: case report and literature review. Pediatr Surg Int. 2007;23(10):1023-6.

4. Cardesa-Salzmann TM, Mora-Graupera J, Claret G, Agut T. Congenital cervical neuroblastoma. Pediatr Blood Cancer. 2004;43(7):785-7.

5. Friedman ER, John SD. Imaging of pediatric neck masses. Radiol Clin North Am. 2011;49(4):617-32.

6. Papaioannou G, McHugh K. Neuroblastoma in childhood: review and radiological findings. Cancer Imaging. 2005;5:116-27.

7. Stern JS, Ginat DT, Nicholas JL, Ryan ME. Imaging of pediatric head and neck masses. Otolaryngol Clin North Am. 2015;48(1):225-46.

8. Díaz-Manzano JA, Pelegrín-Hernández JP, Mínguez-Merlos N, CegarraNavarro MF. Oropharyngeal approach as a surgical alternative for cervical lymphatic malformation with airway compression. Int J Pediatr Otorhinolaryngol. 2014;78(7):1176-8.

9. Elluru RG, Balakrishnan K, Padua HM. Lymphatic malformations: diagnosis and management. Semin Pediatr Surg. 2014;23(4):178-85. 